\title{
Determinantes sociais da saúde uma reflexão sobre a questão social e a contribuição da estratégia de saúde da família
}

\section{Social determinants of health a reflection on the social issue and the contribution of the family health strategy}

\author{
DOI: $10.46814 /$ lajdv3n5-049
}

Recebimento dos originais: 01/05/2021

Aceitação para publicação: 31/06/2021

\section{Luiz Cesar Rodrigues Vieira}

\author{
Maria Angela Boccara Paula
}

\section{RESUMO}

Tendo em vista a aproximação da ESF com o social e a preocupação em promover o acesso a saúde voltando-se prioritariamente para os grupos sociais mais vulneráveis pretende-se com este artigo: discorrer sobre a questão social e sua relação com fenômeno da desigualdade social; identificar a contribuição da Estratégia de Saúde da Família no enfrentamento das desigualdades sociais e sua relação com os determinantes sociais da saúde. Trata-se de uma reflexão teórica sobre a questão social e a Estratégia de Saúde da Família como modelo para redução das iniquidades em saúde. Optou-se por uma revisão de literatura, do tipo narrativa em que os artigos selecionados abordaram os temas: determinantes sociais em saúde, questão social e estratégia de saúde da família. Os resultados apontam que a questão social está intimamente relacionada com capital e as relações sociais impostas pelo mesmo e que tal processo é histórico no que diz respeito ao desenvolvimento da sociedade brasileira. Tendo em vista a iniquidade em saúde como uma das faces da questão social, as políticas de saúde apontam a ESF como uma das formas de enfrentar a desigualdade social dado que, nos determinantes sociais encontramos a expressão da questão social, e com uma prática pautada nos princípios da territorialização, do diagnóstico comunitário, da intersetorialidade e da equidade tal modelo pode ser promissor e resolutivo frente as demandas sociais as quais se relacionam com a saúde.

Palavras-chave: determinantes sociais da saúde, saúde da família, questão social.

\begin{abstract}
Considering the approximation of the FHS to social issues and the concern with promoting access to health care focusing primarily on the most vulnerable social groups, this article aims to discuss the social issue and its relation to the phenomenon of social inequality; to identify the contribution of the Family Health Strategy in combating social inequalities and its relation to the social determinants of health. This is a theoretical reflection on the social issue and the Family Health Strategy as a model for reducing health inequities. We chose a narrative literature review, in which the selected articles addressed the following themes: social determinants of health, social issue, and family health strategy. The results point out that the social issue is closely related to capital and the social relations imposed by it, and that such a process is historical with respect to the development of Brazilian society. In view of the inequity in health as one of the faces of the social issue, health policies point to the ESF as one of the ways to face social inequality given that, in the social determinants we find the expression of the social issue, and with a practice based on the principles of territorialization, community diagnosis, intersectoriality and equity, such model can be promising and resolutive in the face of social demands which are related to health.
\end{abstract}


Keywords: social determinants of health, family health, social issue.

\section{INTRODUÇÃO}

A formulação e a implementação das políticas públicas de saúde no Brasil, resultado da conjuntura sociopolítica onde ao estado é atribuído a responsabilidade de garantia da democracia dos direitos humanos, sociais e de acesso à saúde.

A consolidação do SUS é uma das conquistas populares mais evidentes em que se concretiza com o compromisso do estado em reconhecer a saúde como produção social e reduzir as iniquidades, bem como garantir o direito de acesso universal e igualitário às ações e serviços para promoção, proteção e recuperação da saúde. Sua construção reflete o contexto político de redemocratização do país e a busca pela ampliação dos direitos sociais de acesso à saúde, o qual idealizava o movimento sanitário (RODRIGUES, 2014).

A Constituição Federal do Brasil, aprovada em 1988, propõe no artigo 196 que "a saúde é direito de todos e dever do Estado" e deve tal direito deve ser garantido mediante as políticas sociais e econômicas que visam a redução do risco de doença e agravos, bem como a efetivação de um sistema de saúde universal igualitário e equânime. Parte-se do pressuposto de que as políticas de saúde integram as políticas sociais, assim como o sistema de saúde é um dos componentes dos sistemas de proteção social (PAIM, 2013).

Nessa concepção, fica implícito que nas políticas públicas, a relevância dos determinantes sociais da saúde. Tais fatores são definidos como condições de vida e trabalho dos indivíduos e de grupos populacionais os quais estão relacionados, ou sejam, influenciam a situação de saúde do indivíduo. Para a Comissão Nacional sobre os Determinantes Sociais da Saúde (CNDSS), são exemplos os fatores sociais, econômicos, culturais, étnicos/raciais, psicológicos e comportamentais que influenciam a ocorrência de problemas de saúde e seus fatores de risco na população (BUSS; PELLEGRINI FILHO, 2007).

O conceito de saúde considera não só as causas biológicas da doença, mas também as causas sociais, visto que é resultante das condições de alimentação, habitação, educação, renda, meio ambiente, trabalho, transporte, emprego, lazer, liberdade, acesso e posse da terra e acesso aos serviços de saúde. Sendo assim, é principalmente o resultado das formas de organização social, de produção e podem gerar grandes desigualdades nos níveis de vida (OLIVEIRA e SANTO, 2014).

Infere-se que a saúde possui fatores determinantes os quais resultam da organização social e econômica do país e faz parte das relações sociais as quais devem ser consideradas um processo contínuo e no bojo dessa conjuntura e de todo processo histórico inerente as políticas públicas de saúde, 
é inegável a necessidade de compreender a origem as transformações societárias advindas da expansão capitalista, caracterizada pela globalização da economia culminando na "questão social" (GARBOIS; SODRE; DALBELLO-AAUJO, 2014).

A “Questão Social” é definida como a expressão das desigualdades geradas pelo modo de produção capitalista, e que na relação conflituosa entre o capital e o trabalho surge a relação entre os donos dos meios de produção e aqueles que vendem a sua força de trabalho, e nessa separação divisão de classe, o produz as desigualdades sociais e suas múltiplas expressões da questão social, a exemplo: violência, miséria, desemprego, discriminação, abandono, tráfico de drogas, prostituição, dentre outros. Portanto, a condição social de uma pessoa influencia e determina o ambiente de moradia, de trabalho e a saúde (OLIVEIRA e SANTO, 2014).

À medida que a saúde passa a ser reconhecida como uma produção social, são necessárias múltiplas reformulações no plano da administração e gestão do sistema das práticas assistenciais e de formação do profissional de saúde pública.

É necessário a ampliação do estudo sobre os determinantes do processo saúde-doença e a reformulação de políticas de saúde pública que considerem o social, político e econômica em um mesmo grau de relevância. Exemplo disso é o conjunto de atividades que se estabelece na Estratégia de Saúde da Família (ESF), em que a "práxis" que tem proporcionado aos sujeitos sociais nela envolvidos, conhecer com acuidade, contribuindo de forma efetiva e eficazes o enfrentamento das desigualdades sociais (PERES, 2007).

A ESF é concebida como uma lógica assistencial que visa reverter o modelo biologicista, por meio da mudança no objeto da atenção e na forma de atuação e da organização dos serviços da Atenção Básica, pautado nas ações de Vigilância em Saúde a partir dos determinantes sociais da saúde, como foco na família e nas relações as quais a compõem, aproximando o contexto social do contexto clínico e epidemiológico e promovendo ações intersetoriais. É reconhecida como eixo estruturante do processo de reorganização do Sistema Único de Saúde (SUS) garantindo resolutividade de grande parte dos problemas de saúde da comunidade (FERTONANI, 2015).

Tendo em vista a integração entre as diversas faces das necessidades de saúde, é na formulação das políticas públicas e no campo das práticas de saúde que emerge a necessidade de reflexões sobre o impacto da questão social e sua interface com tal setor. Para tanto, se faz necessário o olhar da equipe de saúde para um diagnóstico de saúde da comunidade onde a "questão social" é elemento relevante na programação das ações das equipes de saúde da família frente as necessidades sociais, situação de vulnerabilidade e iniquidade em saúde (SENNA, 2002).

Nesse sentido, tendo em vista a aproximação da ESF com o social e a preocupação em promover o acesso à saúde voltando-se prioritariamente para os grupos sociais mais vulneráveis, o objetivo desse 
estudo foi discorrer sobre a questão social e sua relação com fenômeno da desigualdade social; identificar a contribuição da Estratégia de Saúde da Família no enfrentamento das desigualdades sociais e dos determinantes sociais da saúde.

\section{MATERIAIS E MÉTODOS}

Trata-se de uma reflexão teórica sobre a Questão Social e a Estratégia de Saúde da Família como modelo para redução das iniquidades em saúde. Optou-se por uma revisão de literatura, do tipo narrativa em base de dados SCIELO e LILACS.

Os artigos selecionados resultaram de uma busca por meio de 03 cruzamentos. $\mathrm{O}$ primeiro com o uso dos termos "determinantes sociais" e "atenção primária", o segundo optou-se por "desigualdade social" e "saúde pública", e o terceiro "desigualdade social” e "saúde coletiva”. Após análise dos artigos repetidos e exclusão dos que não atendiam o objetivo do estudo, reuniu-se 08 artigos dos últimos 10 anos.

Os artigos selecionados abordavam os temas determinantes sociais em saúde, questão social e estratégia de saúde da família. Para enriquecer a discussão, referenciais teóricos do campo das ciências sociais e outros artigos foram utilizados de conforme a subjetividade e interesse de análise do autor.

\section{COMPREENDENDO A QUESTÃO SOCIAL: CONCEITOS ESSENCIAIS}

Ao se discutir a saúde à luz das relações sociais, como uma relação contínua e dinâmica dado o seu caráter interdependente da estrutura social e de seus desdobramentos, infere-se que a saúde e seus determinantes sociais são expressões da questão social, portanto pretende-se aqui, fundamentar o surgimento da questão social como um fenômeno que necessita de compreensão por parte dos profissionais de saúde pública.

Para explicar a "questão social” no Brasil é necessário compreender a singularidade do processo de formação social, para que se ter condições de dimensionar suas particularidades.

As transformações societárias vêm ocorrendo na economia capitalista desde 1970, e nessa conjuntura, a "questão social” permeia as transformações econômicas, políticas e sociais ocorridas na Europa do Século XIX, e inicialmente era considerada um fenômeno social, decorrente do trabalho urbano e da pauperização. Atualmente a "questão social" é a expressão das desigualdades e lutas sociais em suas múltiplas manifestações e de todos os segmentos sociais envolvidos sendo apreendida como o conjunto das expressões das desigualdades da sociedade capitalista (PINHEIRO; DIAS, 2009).

A formação do capitalismo brasileiro é marcada pela permanente exclusão das classes trabalhadoras dos processos políticos decisórios no país, demonstrando a ação dos setores burgueses e do estado de se anteciparem e conduzirem os processos de mudanças, sem provocar alterações 
estruturantes no modelo econômico, no padrão de acumulação e na lógica do poder político (SANTOS, 2012).

O que caracteriza a produção capitalista não é somente a produção e a mais-valia. Ocorrem nesse contexto a produção e a reprodução de relações sociais. Existem sujeitos que podem comprar a mercadoria "força de trabalho" para empregá-la na produção de mercadorias e sujeitos obrigados a vender "força de trabalho", já que esta é o único bem que possuem”. A produção social é cada vez mais coletiva, o trabalho torna-se mais amplamente social, enquanto a apropriação dos seus frutos é privada e monopolizada por parte da sociedade" (IAMAMOTO, 2008).

A relação entre capital e trabalho gera uma tensão entre os donos dos meios de produção e os que vendem a força de trabalho e que buscam a garantia de privilégios econômicos e políticos. É na resultante da separação de classe detentora dos meios de produção e dos que vendem a sua força de trabalho que se produz as desigualdades sociais e suas múltiplas expressões da questão social: violência, miséria, desemprego, discriminação, abandono, tráfico de drogas, prostituição, dentre outros (OLIVEIRA; SANTO, 2013).

A Questão Social é concebida como expressões das desigualdades sociais que são intrínsecas à sociedade capitalista a qual em sua gênese pode ser explicada a partir do movimento de acumulação e reprodução do capital. É marcada pela emersão do pauperismo, pelos processos sócio-políticos e a utilização do espaço público para viabilizar os interesses privados das frações burguesas, atreladas de forma subalterna ao capital internacional, se tornou uma das principais características da realidade política brasileira (SANTOS, 2012).

Em consonância a esse pensamento, Yamamoto (2008) aponta que a questão social não se dissocia do capitalismo e que em uma sociedade burguesa a gênese da questão social deriva do caráter coletivo da produção. Se contrapõe à apropriação privada da própria atividade humana, mas que em tempos de capital fetiche e enfraquecimento da coletividade, a desigualdade social ao lado da pobreza, emerge como a principal marca da questão social.

Lopes (2008) define a pobreza como um desdobramento das relações históricas e estruturais de oposição entre os interesses de classes, um fenômeno econômico que se configura na questão social derivada das relações entre capital $e$ trabalho.

Nessa conjuntura, a desigualdade social é um fenômeno estritamente contraditório, já que a pobreza cresce na razão direta em que aumenta a capacidade da sociedade de produzir riqueza. Quanto mais se desenvolve as forças produtivas, mais aumentava o contingente daqueles que, além de não ter acesso ao que se produz, são despossuídos das condições materiais de vida (SOUZA; SILVA, SILVA, 2013). 
A globalização e o mercado afirmam esse aspecto contrastando a desigual distribuição da riqueza entre grupos e classes sociais nos vários países, que precisam sofrer uma decisiva intervenção da ação do estado e dos governos para controle da desigualdade social (OLIVEIRA; SANTO, 2013).

No Brasil a desigualdade é de base estrutural o quais alguns elementos históricos e societários são destacados por Ianni (1989). Ao longo da história, o intenso desenvolvimento do capitalismo, as migrações internas que atravessavam os campos e cidades, a urbanização e a industrialização que se expande de modo contínuo. Crescem os movimentos populares de protestos e de reivindicações por condições de trabalhos, e gradualmente, as autoridades percebem a desigualdade social como um problema político e não de polícia. Enquanto a economia cresce, o poder estatal fortalece e a massa de trabalhadores padece.

Ianni (1989) ainda aponta a contradição entre o crescimento econômico e o crescimento da pobreza e das desigualdades sociais. Entre 1930 e 1950 o poder público investiu amplamente na expansão e na diversificação da economia, principalmente em insumos necessários para o crescimento industrial. Na década de 40 à década de 80, a economia cresceu quatorze vezes, mas a distribuição permaneceu desigual. Já nesse período, dos $40 \%$ mais pobre detinham 9,7\% da renda total do país enquanto $10 \%$ dos mais ricos detinha $47,9 \%$ da renda nacional.

No início do século XXI, o país ocupa o $9^{\circ}$ lugar em renda per capita, porém caía para o $25^{\circ}$ lugar ao se tratar da proporção de brasileiros que vivem com a pobreza, configurando-se como um país de alta renda e alta pobreza. Enquanto está entre os $10 \%$ dos mais ricos, o Brasil integra a metade mais pobre dos países em desenvolvimento (PERES, 2007).

É possível perceber como o fenômeno da questão social surge em um país capitalista como o Brasil e tendo em vista os diferentes problemas sociais enfrentados, pode-se dizer que a desigualdade social e a pobreza constituem-se na expressão materializada deste fenômeno.

Evidencia-se um quadro amplo para intervenção de políticas sociais articuladas com as políticas macroeconômicas que garantam um crescimento econômico sustentado, a geração de emprego, a elevação da renda proveniente do trabalho e, sobretudo, a redistribuição de renda ainda altamente concentrada no Brasil (SILVA, 2014).

O desafio é a manutenção de níveis significativos e sustentáveis de crescimento econômico; o controle da inflação, o desenvolvimento de serviços de infraestrutura básica com oferta de serviços básicos de forma ampliada e democrática para todo o segmento da sociedade. 


\section{SAÚdE, QUESTÃO SOCIAL E SEUS DETERMINANTE SOCIAIS: REFLEXÕES TEÓRICAS E CONTRIBUIÇÕES DA ESTRATÉGIA DE SAÚDE DA FAMÍLIA.}

Após breve reflexão teórica da questão social e de sua conjuntura, pretende-se aqui problematizar o recente debate advindo do campo dos determinantes sociais da saúde, tendo em vista a necessidade de aprimorar o olhar do profissional da saúde para o "social” e a contribuição da Estratégia de Saúde da Família como modelo que deve reduzir as iniquidades em saúde.

A saúde possui relação estreita com a forma de organização da sociedade e tendo em vista as teorias do processo de saúde doença o que permite aos autores das ciências da saúde definir os determinantes sociais como os fatores que influenciam a ocorrência de problemas de saúde e seus fatores de risco na população e resultam da dinâmica societária. Sendo assim, as condições sociais impostas pelo capital são deletérias à saúde e fazem com que a falta de saúde se configure também como uma condição socialmente imposta. “A própria problemática da saúde-doença ora é fruto direto da acumulação capitalista, ora é condicionada por ela" (SOUZA; SILVA; SILVA, 2013).

As políticas públicas de saúde, estão pautadas no direito de todos à saúde e no dever do estado em garantir a saúde mediante políticas sociais e econômicas para superar as iniquidades e o acesso ao serviço com qualidade. No entanto, se contrapondo ao que se idealiza na constituinte e ao que se propõe na lei 8080/90, um dos principais desafios é a garantia dos princípios norteadores da universalidade e da equidade, ambos amplamente fragilizados em diversos segmentos da sociedade, prejudicando o acesso e limitado pelas desigualdades sociais (RODRIGUES, 2014).

Uma das formas de reduzir as desigualdades sociais e atuar de forma eficiente como foco nos determinantes sociais da saúde, é a implementação de modelos de atenção à saúde que favorecessem, ao mesmo tempo, a equidade, a integralidade e a universalidade e que as práticas sejam orientadas pelas necessidades de saúde das pessoas em aproximação com o social.

Recém aprovada, apesar de diferentes opiniões de entidades importantes no campo da saúde coletiva no que diz respeito as mudanças propostas, a nova Política Nacional de Atenção Básica obteve sua legalidade pela portaria $\mathrm{n}^{\circ} 2488$, estabelecendo a revisão de diretrizes e normas para a organização da atenção básica, para a Estratégia Saúde da Família (ESF). Apesar de críticas contundentes, as quais não serão aqui, objeto de análise, parte-se da premissa que a essência e o significado da atenção básica foram mantidos (BRASIL, 2017)

A Atenção Básica deve ser ofertada integralmente e gratuitamente a todas as pessoas de acordo com suas necessidades e demandas do território, considerando os determinantes e condicionantes da saúde; proibida qualquer exclusão baseada em idade, gênero, raça/cor, etnia, crença, nacionalidade, orientação sexual, identidade de gênero, estado de saúde, condição socioeconômica, escolaridade, limitação física, intelectual, funcional e outras, com estratégias que permitam minimizar desigualdades/iniquidades, evitem exclusão social de grupos que 
sofram estigmatização ou discriminação, de maneira que impacte na autonomia e na situação de saúde.

A ESF tem como princípio norteador a vigilância em saúde, a prevenção de doenças e a promoção da saúde, além de ser resolutivo para problemas de baixa complexidade clínica. Assume-se um caráter substitutivo em relação à "atenção básica tradicional” uma vez que, adota princípios norteadores, entre os quais destacam-se a adstrição de clientela, a territorialização, o diagnóstico da situação de saúde da população e o planejamento baseado na realidade local (FERTONANI, 2015).

Esses princípios favorecem o reconhecimento das variáveis sociodemográficas da comunidade, e permite o planejamento de ações que atendam às necessidades impostas pela desigualdade social e iniquidade em saúde.

Mediante tais características, espera-se que as ações sejam pautadas na coletividade, centrada no território, por meio de uma prática transformadora em que a saúde e o contexto social sejam o foco, e não somente a doença. Assume-se uma prática intersetorial que ultrapassa o setor saúde e se aproxima dos determinantes sociais do processo saúde e doença (DOWBOR; WHESTPHAL, 2013).

A discussão sobre o "social” compõe a agenda política do setor saúde quando a Organização Mundial da Saúde (OMS), e como resultado disso, cria em 2005, a Comissão sobre os Determinantes Sociais da Saúde (CDSS). Tal comissão é incumbida em difundir, em âmbito internacional, o reconhecimento sobre a importância dos determinantes sociais na situação de saúde de indivíduos e sobre a necessidade de enfrentar as desigualdades por eles geradas (GARBOIS; SODRE; DALBELLOAAUJO, 2014).

Em março de 2006 foi criada no Brasil a Comissão Nacional sobre os Determinantes Sociais da Saúde (CNDSS), a qual trouxe para a agenda política brasileira a discussão, já iniciada, sobre a necessidade de intervir nos determinantes sociais na busca pela equidade em saúde (BRASIL, 2006).

De acordo com a CNDSS, tendo em vista as causas sociais das iniquidades em saúde, é recomendado que a atuação sobre os determinantes sociais paute-se em três pilares fundamentais: ações intersetoriais que visem a qualidade de vida e a utilização de recursos da comunidade, a participação social e a promoção da autonomia dos grupos mais vulneráveis da população; evidências científicas, que incorpore a produção sistemática de informações e conhecimentos sobre as relações entre os determinantes sociais, a saúde e a avaliação das intervenções produzidas (GARBOIS; SODRE; DALBELLO-AAUJO, 2014).

Dos três pilares, dois deles são princípios da ESF. A intersetorialidade e o controle social e no que diz respeito as evidências científicas, é no campo da saúde coletiva que se concentram o maior número pesquisas sobre as questões referentes aos determinantes sociais. 
Se o capitalismo e a globalização da economia possuem parcela de responsabilidade na constituição da questão social, os determinantes sociais da saúde são produtos dessa relação. A vulnerabilidade aumenta em condições desfavoráveis do ponto de vista socioeconômico. A mortalidade de crianças menores de 5 anos, a desnutrição em mulheres, assim como, a proporção de crianças com atraso no crescimento era 3,2 vezes mais alta nos países pobres. Em se tratando de Brasil, "a mortalidade infantil está relacionada com a renda das famílias, o nível de educação da mãe, as condições do domicílio, o local em que vive e a situação social da família da criança. (BUSS, 2007).

Muito embora os indicadores de desigualdades sociais na área da saúde sejam muitos, a ESF, desde sua implementação, estabeleceu como uma de suas prioridades reduzir as taxas de mortalidade materna e infantil.

O Ministério da Saúde realizou em 2005 um estudo para avaliar a contribuição da ESF na redução da Taxa de Mortalidade Infantil (TMI) no Brasil no período de 1990 a 2002. Para cada 10\% de aumento na cobertura populacional do PSF há uma redução média de $4.6 \%$ da taxa de moralidade infantil. Para se ter ideia da eficácia das ações nesse indicador, ele acaba por ser mais eficaz na redução da TMI quando comparado com a eficácia da ampliação da rede de esgoto e do número de leitos hospitalares infantis, o que reduz respectivamente a TMI em $3,2 \%$ e $1,4 \%$.

Esse mesmo estudo aponta a importância dos aspectos sociais. A relevância do Programa Saúde da Família na queda da TMI, no Brasil, só não é maior do que a variável "acesso das mulheres à educação", em especial a alfabetização das mesmas. Nesse caso, uma ampliação de $10 \%$ de cobertura significa diminuir em 16.8\% a mortalidade das crianças com até um ano de idade (PERES, 2007).

$\mathrm{Na}$ opinião de gestores e profissionais das equipes de saúde com a implantação da ESF, houve melhora no acesso da população ao sistema de saúde, maior o acesso de camadas de população de baixa renda com as ações de territorialização e com o papel dos agentes comunitários de saúde. Com isso, indiretamente, proporcionou-se certa igualdade na efetividade dos serviços, ao promover uma qualificação das práticas baseada em um conjunto novo de valores, finalidades e sentidos, avaliados do ponto de vista da realização de resultados na direção efetiva dos usuários, aproximando o biológico do subjetivo, ampliando o objeto de trabalho (SISSON, 2007).

Apesar dos avanços e da inegável contribuição da ESF em relação à condição de saúde da população, não só pelos preceitos teóricos, mas pela redução dos indicadores de saúde, torna-se necessário ressaltar que o desafio do acesso à saúde, o modelo medicocêntrico e pautado na doença e os interesses políticos da gestão local podem contribuir negativamente para o insucesso dessa estratégia. 


\section{CONCLUSÃO}

Diante do que foi demonstrado, o conjunto dos problemas sociais que se enfrenta atualmente na sociedade possui visivelmente uma base material composta por uma tensão entre a classe dominante e a classe desfavorecida. No bojo dessa relação, a saúde é o resultado da forma que a sociedade está organizada, e podemos atribuir que a determinação social da saúde é essencialmente econômica.

A formulação de políticas de combate às iniquidades sociais ocorre em uma conjuntura políticoeconômica, os quais, os interesses de expansão do capital se situam nas entrelinhas dos objetivos de promoção à justiça social. Surge a partir desse cenário, uma demanda social a ser priorizada aos atores envolvidos no setor saúde, tais como o estado com a formulação de políticas e os profissionais de saúde com as intervenções nesse campo.

A contribuição da ESF como estratégia que reúne as ferramentas necessárias para intervir no processo saúde doença com destaque a intersetorialidade, a abordagem da coletividade e o diagnóstico comunitário pautado em determinantes sociais da clientela adscrita. A mudança nas práticas não deve ser apenas normativa, em cumprimento aos preceitos do programa, e sim, uma mudança de comportamento do profissional que assume o compromisso em reconhecer o indivíduo em sua totalidade.

Nesse contexto é possível afirmar que a qualificação dos atores estratégicos (profissionais) do SUS e na atenção básica devem estar aptos a compreender e entender o cotidiano de vida do indivíduo, para então, implementar ações resolutivas de forma a permitir a intervenção DSS. Refletir sobre o campo de produção do conhecimento e de práticas em saúde, de modo a problematizar a situação dos determinantes sociais, para além de indicadores, fatores e causalidades pode ser um dos caminhos para uma assistência justa e equânime. 


\section{REFERÊNCIAS}

DOWBOR, Tatiana Pluciennik; WESTPHAL, Marcia Faria. Determinantes sociais da saúde e o Programa Saúde da Família no Município de São Paulo. Rev. Saúde Pública, São Paulo, v. 47, n. 4, p. 781-790, ago. 2013 .

BUSS, Paulo Marchiori. Globalização, pobreza e saúde. Ciênc. Saúde coletiva, Rio de Janeiro, v. 12, n. 6, p. 1575-1589, Dec. 2007.

BUSS, Paulo Marchiori; PELLEGRINI FILHO, Alberto. A saúde e seus determinantes sociais. Physis: Rev. Saúde Coletiva, Rio de Janeiro, v. 17, n. 1, p. 77-93, 2007. Disponível em: <http://www.scielo.br/pdf/physis/v17n1/v17n1a06.pdf>. Acesso em: 10 de dezembro de 2017.

SILVA, Maria Ozanira da Silva e. Pobreza, desigualdade e políticas públicas: caracterizando e problematizando a realidade brasileira. Rev. Katálysis, Florianópolis, v. 13, n. 2, p. 155-163, 2010.

FERTONANI, Hosanna Pattrig et al. Modelo assistencial em saúde: conceitos e desafios para a atenção básica brasileira. Ciência \& Saúde Coletiva, [s.1.], v. 20, n. 6, p.1869-1878, Jun. 2015.

GARBOIS, Júlia Arêas; SODRE, Francis; DALBELLO-ARAUJO, Maristela. Determinantes sociais da saúde: o "social”" em questão. Saúde soc., São Paulo, v. 23, n. 4, p. 1173-1182, dezembro de 2014.

IAMAMOTO, M.V. Estado, classes trabalhadores e política social no Brasil. In. BOSCHETTI, I. et al. (Org.) Política Social no Capitalismo: tendências contemporâneas. São Paulo: Cortez, 2008, p. $13-43$.

IANNI, Octávio. “O Brasil Moderno”. In: A ideia de Brasil Moderno. 2a ed., São Paulo: Brasiliense: 1994. p.7-50

LOPES, J. R. Processos sociais de exclusão e políticas públicas de enfrentamento da pobreza. Cadernos CRH [online]. 2008, vol.21, n.53, p.347-360. Disponível em http://www.scielo.br/scielo.php?pid=S0103-49792008000200011\&script=sci_abstract\&tlng=pt.

Acesso em 13 dezembro de 2017.

NETTO, José Paulo. Economia política: uma introdução crítica, São Paulo: Cortez, 2006. PELLEGRINI FILHO, Alberto. Compromisso com a ação. Radis, $n^{\circ}$ 47, julho 2006. Disponível em: http://www.determinantes.fiocruz.br/compromissocomacao.htm. Acesso em 10 de dezembro de 2017

OLIVEIRA MJI, SANTO EE. A relação entre os determinantes sociais da saúde e a questão social. Caderno Saúde e Desenvolvimento. v.2 n.2, p.7-24, 2013.

PAIM Jair Nilson et al., O sistema de saúde brasileiro: história, avanços e desafios. Série: Saúde no Brasil. $\quad$ p. $11-31, \quad 2011 . \quad$ Disponível em: http://download.thelancet.com/flatcontentassets/pdfs/brazil/brazilpor1.pdf. Acesso em 10 de dezembro de 2017.

PERES, Ellen M., O Programa Saúde Da Família no enfrentamento das desigualdades sociais. Aquilham v. 7, n. 1, p. 64-76, 2007. Disponível em http://www.redalyc.org/articulo.oa?id=74170106. Acesso em 10 de dezembro de 2017. 
PINHEIRO, Farias; DIAS, Oliveira. Questão Social: um conceito revisitado, Contribuciones a las Ciencias Sociales, Fevereiro, 2009, Disponível em www.eumed.net/rev /cccss/03/fpod.htm. Acesso em 08 de Dezembro de 2017.

RODRIGUES, Paulo Henrique de Almeida. Desafios políticos para a consolidação do Sistema Único de Saúde: uma abordagem histórica. Hist. cienc. Saúde - Manguinhos, Rio de Janeiro, v. 21, n. 1, p. 37-60, mar. 2014 . Disponível em http://www.scielo.br/scielo.php?script=sci_arttext\&pid=S0104 $59702014000100037 \& \operatorname{lng}=$ en\&nrm=iso. Acesso em 18 de Novembro de 2017.

SANTOS, Josiane Soares. Modos de produção, formação social e alguns marcos históricos sobre o Brasil. In. SANTOS, Josiane. Soares. "Questão Social": particularidades no Brasil. São Paulo: Cortez, 2012.

SENNA, Mônica de Castro Maia. Eqüidade e política de saúde: algumas reflexões sobre o Programa Saúde da Família. Cad. Saúde Pública, Rio de Janeiro, v. 18, supl. p. S203-S211, 2002.

SOUZA, Diego de Oliveira; SILVA, Sóstenes Ericson Vicente da; SILVA, Neuzianne de Oliveira. Determinantes Sociais da Saúde: reflexões a partir das raízes da "questão social". Saude e soc., São Paulo, v. 22, n. 1, p. 44-56, Mar. 2013.

BRASIL. Decreto, de 13 março de 2006. Institui, no âmbito do Ministério da Saúde, a Comissão sobre Determinantes Sociais da Saúde (CNDSS). Diário Oficial da União, Brasília, DF, 14 mar. 2006. Disponível em: <http://www.determinantes.fiocruz.br/decreto.htm>. Acesso em: 09 de set. 2017. 